\title{
Fatigue-related risk management in the emergency department: a focus-group study
}

\author{
Pierre Bérastégui $^{1}\left[\right.$ [ $\cdot$ Mathieu Jaspar ${ }^{1} \cdot$ Alexandre Ghuysen $^{2} \cdot$ Anne-Sophie Nyssen $^{1}$
}

Received: 5 January 2018 / Accepted: 6 May 2018 / Published online: 18 May 2018

(c) SIMI 2018

\begin{abstract}
Fatigue has major implications on both patient safety and healthcare practitioner's well-being. Traditionally, two approaches can be used to reduce fatigue-related risk: reducing the likelihood of a fatigued operator working (i.e. fatigue reduction), or reducing the likelihood that a fatigued operator will make an error (i.e. fatigue proofing). Recent progress mainly focussed on fatigue reduction strategies such as reducing work hours. Yet it has to be recognized that such approach has not wholly overcome the experience of fatigue. Our purpose is to investigate individual proofing and reduction strategies used by emergency physicians to manage fatigue-related risk. 25 emergency physicians were recruited for the study. Four focus groups were formed which consisted of an average of six individuals. Qualitative data were collected using a semi-structured discussion guide unfolding in two parts. First, the participants were asked to describe how on-the-job fatigue affected their efficiency at work. A mind map was progressively drawn based upon the participants' perceived effects of fatigue. Second, participants were asked to describe any strategies they personally used to cope with these effects. We used inductive qualitative content analysis to reveal content themes for both fatigue effects and strategies. Emergency physicians reported 28 fatigue effects, 12 reduction strategies and 21 proofing strategies. Content analysis yielded a further classification of proofing strategies into self-regulation, task re-allocation and error monitoring strategies. There is significant potential for the development of more formal processes based on physicians' informal strategies.
\end{abstract}

Keywords Risk management $\cdot$ Fatigue proofing strategies $\cdot$ Emergency service

\section{Introduction}

Fatigue, defined as a biological drive for recuperative rest [1], has major implications on both patient safety and the well-being of healthcare practitioners. Fatigue has been associated with an increased risk of injury, medical error

Pierre Bérastégui

p.berastegui@ulg.ac.be

Mathieu Jaspar

mathieu.jaspar@ulg.ac.be

Alexandre Ghuysen

a.ghuysen@chu.ulg.ac.be

Anne-Sophie Nyssen

asnyssen@ulg.ac.be

1 Cognitive Ergonomics Laboratory (LECIT), Department of Work Psychology, University of Liège, Sart-Tilman B31, 4000 Liege, Belgium

2 Emergency Department, University Hospital Centre of Liège, Liege, Belgium or adverse events [2] and a decreased quality of life [3]. Physicians working in the Emergency Departments (EDS) are particularly vulnerable to fatigue-related risk. First, emergency physicians (Eps) are regularly asked to work more than $10 \mathrm{~h}$ a day on varying shifts with short breaks to recuperate. The effect of sleep loss is cumulative and can lead to chronic sleep deprivation over months or years [4]. Second, emergency medical practice requires physicians to provide critical care during both the day and night. Working at night generates persistent conflicts with the biological clock, and rotating shifts prevent circadian adjustment to a new sleep-wake cycle from taking place [5].

Recent approaches to address fatigue-related risk have mainly focussed on reducing working hours, and ensuring sufficient recuperation time. The limitations of such approaches have been demonstrated with an increase in the number of visits to the ED, and a shortage of Eps. In 2003, the European Parliament authorised an additional weekly working time of $12 \mathrm{~h}$ for emergency physicians to ensure continuity of care, increasing the weekly working time to 
$72 \mathrm{~h}$ a week. Additionally, the daily working time has been raised to $24 \mathrm{~h}$ followed by at least $12 \mathrm{~h}$ of rest. In this context, situations where fatigued physicians are working continue to arise frequently. Handel et al. show that $38 \%$ of 602 Eps completing the Epworth Sleepiness Scale suffer from excessive daytime sleepiness [6]. More recently, another study, using the Pittsburgh Sleep Quality Index, involving 37 Eps reports that $81 \%$ experience poor sleep quality [7].

Dawson and McCulloch first introduced the notion of fatigue proofing as a complementary approach to fatigue reduction [8]. Fatigue Proofing Strategies (FPS) aim to reduce the likelihood that a fatigued individual operating in the workplace will make an error. In contrast, Fatigue Reduction Strategies (FRS) aim to reduce the likelihood of a fatigued individual operating in the workplace. These two sets of strategies correspond to different levels of control. FRS are achieved through organisational controls such as the prescription of maximum shift and minimum break duration, while FPS typically develop as informal work practices within a work group. Consequently, most formal risk-control systems do not encompass the notion of fatigue proofing and FPS constitute hidden knowledge for safety managers. According to Dawson and McCulloch, an effective Fatigue Risk Management System (FRMS) requires the systematic identification and formalization of proofing strategies as a complementary approach to fatigue reduction [9].

The effective use of reduction and proofing strategies implies that operators are able to assess their own levels of impaired alertness, and are aware of the detrimental effects of fatigue on performance. Following from this assumption, we aim to: (1) explore Eps' experience of fatigue-related impairments and (2) identify informal reduction and proofing strategies used by physicians to manage fatigue-related risk. Such results may provide useful insights for the development of formal processes and have implications for residency training.

\section{Methods}

\section{Context}

The study took place at the Emergency Department (ED) of the Liège University Hospital Centre in Belgium. This ED handles more than 42,000 patient admissions per year, with an overall department hospital admission rate of $30 \%$. At the time of the study, the ED had 32 Eps. The day shift runs from 8 am to $6 \mathrm{pm}$, while the night shift begins at $6 \mathrm{pm}$ and runs up to the following morning. Emergency physicians are regularly asked to work both shifts in a row, representing a total of $24 \mathrm{~h}$ of service. The current training programme does not include sleep hygiene or other concepts relating to fatigue management.

\section{Sample}

25 Eps agreed to take part in the study (participation rate of $78 \%$ ), including 14 postgraduate trainees (i.e. physicians currently completing a 6-year postgraduate training qualification) and 11 specialists (i.e. physicians who had completed the 6-year postgraduate training qualification).

\section{Data collection}

We created four focus groups with an average of six participants in each. Two groups consisted of postgraduate trainees and the other two involved trained specialists. Participants were asked to describe how on-the-job fatigue affected their efficiency at work. Using XMind 7 software, a mind map was progressively drawn in real-time based upon participants' perceptions of the effects of fatigue. Participants were then asked to describe strategies they personally used on the job to cope with any of these effects. The mind map was only used as a facilitator to begin eliciting strategies. The stop rule was based upon theoretical saturation (i.e. when no new information was forthcoming).

\section{Data analysis}

The data that were analyzed included transcripts of the audio-recorded sessions and field notes written by the interviewer. Data analysis was conducted using inductive qualitative content analysis, allowing categories to emerge directly from the material without any theoretical restrictions. We followed the iterative data analysis steps described in Fig. 1 [10].

Only the material that was relevant to our research aims was considered for analysis. We used the following criteria for data inclusion: verbalisations concerning the perceived effects of fatigue and strategies used to manage fatiguerelated risk. Both the effects and strategies had to be personally experienced on the job and reported by at least one of the session's members.

Open coding was assured by an iterative process of reading through transcripts to create categories for chunks of data. A category was labelled once the material fitted the category formation criteria. Data falling under a previous category were subsumed under that category; otherwise a new category was created. Sub-categories with similar events and incidents were grouped together as categories, and categories were grouped together as main categories. The abstraction process continued as far as it was reasonable and possible. The category system was revised when no more categories were to be found. Duplicates and redundancies were removed to obtain a set of unique elements 


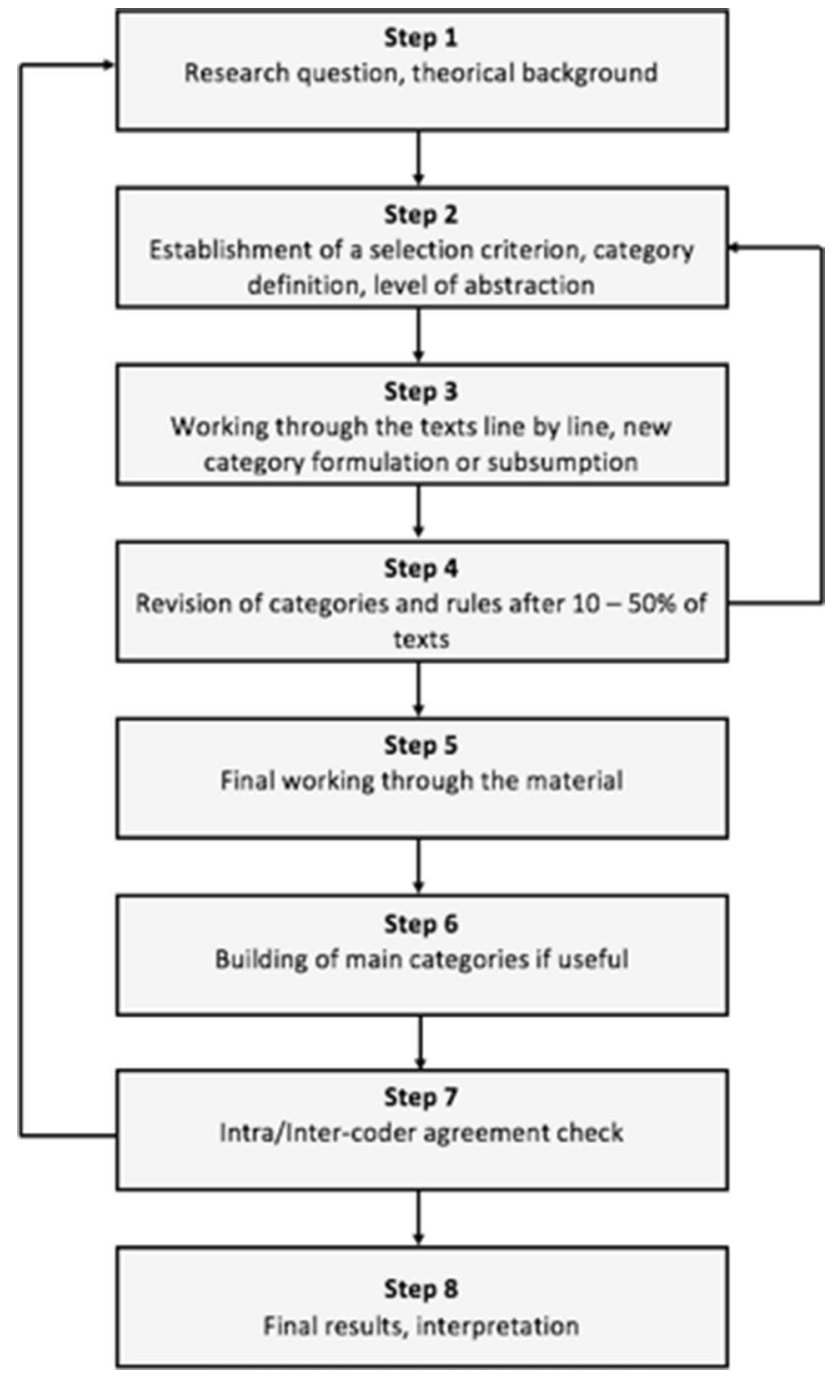

Fig. 1 Inductive content analysis procedure, adapted from Mayring, 2015

under each category. We then checked for category overlaps, proper levels of abstraction and clear category definitions. The entire dataset was worked through again after any changes in the category system. Data analysis resumed when the category system was considered to be valid. Data analysis was carried out by two researchers, comparing categories and discussing inconsistencies until agreement was reached.

\section{Ethical considerations}

The study was approved by the Ethics Committee of the Faculty of Psychology of the University of Liège and by the Ethics Committee of the Medical Centre of the University of Liège. In accordance with the Declaration of Helsinki, all participants gave their written informed consent prior to their inclusion in the study.

\section{Results}

\section{Physicians' experience of fatigue-related impairments}

Participants reported a total of 78 effects of fatigue. We removed duplicates and redundancies, and obtained a set of 28 specific elements (c.f. Fig. 2). Content analysis yielded a two-level categorization. The first level comprises behavioural symptoms $(n=16)$, work performance impairments $(n=6)$ and fatigue-related errors $(n=6)$. The second level further splits behavioural symptoms into specific collections of symptoms: physical $(n=4)$, cognitive $(n=4)$, emotional $(n=5)$ and motivational symptoms $(n=3)$.

Regarding behavioural symptoms, physicians reported physical symptoms such as difficulty staying awake, recurrent headaches associated with noise sensitivity and muscles twitching while performing precise gestures. Visual disturbances were also reported, such as blurred vision, itchy eyes, the inability to judge distance and tunnel vision. Participants also reported cognitive impairments such as being easily distracted by surroundings, resulting in difficulties focussing on a task. Fatigue was also reported as making it difficult to remember things. Other cognitive symptoms included psychomotor impairments resulting in slower gestures and lack of accuracy, as well as decreased decision-making and critical-thinking skills. Physicians reported harshness and irritability issuesbeing edgy and moody - which caused them to easily lose their temper. Fatigue was also associated with difficulties feeling empathy for patients and their families. Participants admitted to possibly using an inappropriate tone or sarcasm. Fatigue was also reported to decrease commitment to the job. Fatigued physicians tend to not engage in proactive behaviours and to focus on first-cause admission. They are less-willing to accept compromises of any kind with patients, families or colleagues. Physicians reported rushing patient interactions such as case history or breaking bad news.

The discussion progressively focussed on how these behavioural symptoms impact upon work performance. According to physicians, communications within teams or with patients and their families are affected by fatigue. Interpersonal conflicts with nurses or peers are more frequent, and physicians tend to avoid social interactions. Physicians admit lacking a critical approach to specialists' reports and to taking fellow physicians' diagnoses at face value. A reduction in communication was assumed to lead to discontinuities in care coordination and a deterioration of the overall vision of the ED. Physicians reported difficulties in accurately anticipating how patients' 
Fig. 2 Reported effects of fatigue-results from content analysis

\begin{tabular}{|l|l|}
\hline Physical \\
\hline Difficulty to stay awake \\
\hline Muscle twitching \\
\hline Headache \\
\hline Visual disturbances \\
\hline Cognition \\
\hline Difficulty to stay focus \\
\hline Difficulty to remember things \\
\hline Gesture slowing and lack of accuracy \\
\hline Decrease quality of decision and critical thinking \\
\hline Emotion \\
\hline Harshness \\
\hline Irritability \\
\hline Lack of empathy \\
\hline Disinhibition \\
\hline Motivation \\
\hline Lack of proactivity \\
\hline Negligence \\
\hline Lack of flexibility \\
\hline Hastiness \\
\hline Resident productivity \\
\hline Patient monitoring \\
\hline Team communication \\
\hline Patient communication \\
\hline Care coordination \\
\hline Situational awareness \\
\hline Incomplete records \\
\hline Unnecessary testing \\
\hline Inappropriate drug regimen \\
\hline Cases muddle up \\
\hline Omission \\
\hline Fixation \\
\hline
\end{tabular}

conditions would develop. Fatigue was also reported to globally decrease productivity, defined as the number of cases handled over the course of the shift.

Physicians reported specific errors to illustrate the operational consequences of these work performance impairments. Unnecessary testing and overprescription were typical examples of team communication and care discontinuity issues. Physicians reported mixing up information between cases, which ultimately affected the accuracy of medical records and diagnoses. Generic failures, such as omission or fixation errors, were also reported as common fatigue-related errors.

\section{Informal fatigue management strategies}

Physicians reported 58 fatigue management strategies across focus groups. We removed duplicates and redundancies to obtain a set of 12 fatigue reduction strategies and 21 fatigue proofing strategies (c.f. Fig. 3).

\section{Fatigue reduction strategies}

Physicians reported using physiological refreshments such as food or energy drinks, physical exercise, getting fresh air or freshening up with cold water $(n=7)$ to reduce subjective feelings of sleepiness. Only two strategies related to resttime management: having a nap and re-grouping tasks to maximise rest time. Physicians also reported reducing subjective feelings of fatigue through social interactions $(n=1)$ and listening or thinking about music $(n=2)$.

\section{Fatigue proofing strategies}

Content analysis yielded three sub-categories of FPS: selfregulation, task re-allocation and error monitoring strategies.

Self-regulation strategies $(n=14)$ aim to compensate for the behavioural symptoms associated with fatigue to sustain acceptable work performance. Reported strategies include the use of cognitive aids, self-motivation and seeking emotional or instrumental support. For instance, 
Fig. 3 Reported fatigue management strategies-results from content analysis

\begin{tabular}{|c|c|c|}
\hline \multicolumn{3}{|c|}{ Figure 2: fatigue management strategies } \\
\hline \multirow{12}{*}{ 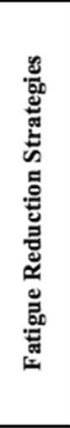 } & \multirow{12}{*}{ Fatigue Reduction } & I have a quick snack \\
\hline & & I sing, I hum or I think about songs \\
\hline & & I have an energy drink (coffee, tea, coke, etc.) \\
\hline & & I listen to music while doing paperwork to stay awake \\
\hline & & I have a nap whenever it is possible \\
\hline & & I try to keep moving as much as possible to stay awake \\
\hline & & I try to anticipate and regroup tasks as much as possible in order to maximize rest time \\
\hline & & I take advantage of lull periods to have a non-work related talk with my colleagues \\
\hline & & I take a brief shower or I dampen my face with cold water \\
\hline & & I take advantage of lull periods to take the time to eat slowly \\
\hline & & I take advantage of lull periods to be physically active (running, walking, etc.) \\
\hline & & I take advantage of lull periods to take some fresh air and/or to smoke a cigarette \\
\hline \multirow{21}{*}{ 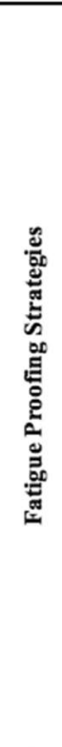 } & \multirow{13}{*}{ Self- Regulation } & I engage conversation with the patient on a humorous note \\
\hline & & I do some self-motivation, I tell myself to hold on and that the shift is about to end \\
\hline & & I seek moral support from my colleagues \\
\hline & & I complete the patient record as and when it comes rather than letting things pile up \\
\hline & & I use algorithms or other cognitive aids more often \\
\hline & & I write down things I've left to do so I don't forget them \\
\hline & & I take a brief moment to relieve the tension before interacting with a patient \\
\hline & & I tend to interact with patient in way that creates a climate of closeness \\
\hline & & I try to vary tasks as much as possible to avoid boredom \\
\hline & & I strongly focus to avoid any inattention mistakes \\
\hline & & I rely more on manuals or databases than my memory \\
\hline & & I focus on one task at a time \\
\hline & & I communicate more with my colleagues \\
\hline & \multirow{4}{*}{ Task Reallocation } & I seek instrumental support from my supervisor or colleagues \\
\hline & & I defer secondary goals to colleagues while I take care of the primary goal \\
\hline & & I rely more on experimented nurses for specific tasks they are qualified for \\
\hline & & I defer complex but non-urgent tasks to my colleagues working the following shift \\
\hline & \multirow{4}{*}{ Error Monitoring } & I double check drug prescriptions and dosage regimen \\
\hline & & I talk to myself or others about what I've done to make sure I didn't forget anything \\
\hline & & I let nurses know I'm tired so they pay more attention to what I'm doing \\
\hline & & I ask a colleague to double-check what I've done \\
\hline
\end{tabular}

physicians complete the patient records as and when it comes, rather than letting things pile up to compensate for the impact of fatigue on short-term memory. Physicians also tend to interact with patients in a way that creates a climate of closeness, and to inject a humorous note into conversations to compensate for irritability or harshness.

Task re-allocation strategies $(n=4)$ aim to redistribute the tasks within the team to minimize error opportunities. These strategies are team-based adaptations leading to a better match between operators' resources and task constraints. For instance, physicians reported deferring complex tasks during the night shift to colleagues working the following morning. Physicians also admitted to relying more heavily on experienced nurses for specific tasks it is assumed they are qualified to perform.

Error monitoring strategies $(n=4)$ aim to prevent fatigue-related errors from developing into adverse events. These strategies form the last layer, preventing the hazard from turning into a fatigue-related incident. For instance, physicians reported double-checking, either themselves or using others, for tasks that they assume are sensitive to fatigue-related risk. Physicians also reported verbalizing acts or prescriptions to make sure they do not forget anything.

\section{Discussion}

According to Dawson et al., the effective management of fatigue-related risk requires the use of fatigue proofing as a complementary approach to fatigue reduction [9]. However, FPS typically develop as implicit elements of the safety system and most formal controls do not encompass the notion of proofing. It is a challenge to identify these strategies at a local level to investigate their potential for further formalisation. On the other hand, the effective use of fatigue management strategies implies that operators are able to recognise the early signs of fatigue and can anticipate its effects on work performance. Following this, our aim was: (1) to investigate physicians' experience of fatigue-related impairments and (2) to identify informal strategies used by physicians to manage fatigue-related risk. 


\section{Emergency physicians' experience of fatigue-related impairments}

Our results show that physicians experience a wide variety of fatigue-related impairments. Physicians reported behavioural symptoms, work performance impairments and fatigue-related errors.

Studies conducted in controlled settings repeatedly demonstrated that fatigue reduces cognitive processing speed, and therefore, increase inattentiveness and tunnel vision [11]. Fatigue is also known to impair brain areas involved in working memory [12], visuomotor performance [13] and decision-making [14]. Psychomotor skills are less affected than cognitive skills, but poor cognitive performance may also lead to limited psychomotor proficiency [15]. Prolonged fatigue has also been associated with physical symptoms such as recurrent headaches, muscle pain, sleeping during the day and visual disturbances [16]. The emotional dimension of fatigue is known as Compassion Fatigue (CF) and is caused by prolonged, continuous and intense exposure to patients' traumatic experiences [17]. CF is characterized by anger, irritability, lower work satisfaction and a reduced ability to feel sympathy and empathy. It also appears that sleep deprivation could lead to a loss of motivation and task-directed efforts [13]. Our results show that physicians' experience of behavioural symptoms associated with fatigue is consistent with the literature. Emergency physicians are aware of the wide array of fatigue symptoms and reported symptoms that covered the physical, emotional, motivational and cognitive dimensions of fatigue.

Work performance impairments reported by physicians are also consistent with studies conducted in residency settings. The literature supports physicians' perceived effects of fatigue on productivity [18], situation awareness [19], patient-centred communications [20] and teamwork [21]. Extended periods of time in the ED have been demonstrated to be strongly, negatively connected to physicians' patientsper-hour rate [18]. Emotional exhaustion is associated with a reduction in empathy and compromises the quality of interactions with patients [20]. Interactions with colleagues are also impacted and Singh and colleagues demonstrated that a breakdown in teamwork is a factor in $70 \%$ of the cases resulting in patient injury [22].

Finally, physicians reported fatigue-related errors regularly occurring in ED. Fatigue is known to be associated with an increased risk of medical error [23]. However, few studies have attempted to describe the nature of fatiguerelated errors in residency settings. Our results show that several reported errors could be attributed to the way physicians use and manage clinical data. Physicians reported experiencing difficulties maintaining complete and up-todate medical records and confusing clinical data between multiple cases. The failure to revise situation assessments as new evidence comes in (e.g. fixation errors) ordering unnecessary diagnostic tests, and delivering inappropriate drug regimens were also reported as typical fatigue-related errors. This is particularly relevant, considering that $80 \%$ of the medical errors involve informational or personal miscommunication [24], and that fatigue is estimated to be a contributing factor in $65 \%$ of these cases [25].

Taken together, these results show that physicians are aware of the detrimental effect of fatigue on human functioning and how these symptoms are reflected in the work environment. However, do they use this knowledge to manage fatigue-related risk?

\section{Fatigue reduction strategies}

Physicians reported 12 unique FRS across the focus group sessions. Only two reported strategies aim to rationalize rest time. Physicians reported napping for up to $4 \mathrm{~h}$ and re-grouping, anticipating or delaying tasks to maximise the length of the nap. They used the term 'nap' to refer to both short recuperative rest and longer sleep periods. Research has shown that short naps of between 10 and 20 min significantly improve alertness [26], but longer naps cause significant sleep inertia [27]. Sleep inertia is associated with decreased cognitive performance, irritability, confusion and automatic behaviours [28] and represent a significant risk in terms of occupational safety. Caffeine consumption directly preceding a short nap (i.e. powernaps) has been demonstrated to be an effective way of reducing the sleep inertia period [29]. In addition, participants reported napping exclusively during the night shift. Research shows that strategic napping during the day can effectively counteract night-time sleepiness [30]. These results highlight the fact that physicians are not well-educated about sleep physiology and stress the need for formal training on how and when to nap.

Other reported strategies include physiological refreshments such as food or energy drinks, physical exercise, getting fresh air or freshening up with cold water. There is evidence in the literature that dehydration and reduced glucose levels are associated with impaired decision-making [31]. Short breaks in natural light for fresh air and meditation are also techniques which are recommended to reduce subjective feelings of sleepiness [32], but lack scientific evidence. Hayashi and Hori investigated face washing as a sleep inertia countermeasure following a nap and showed a temporary reduction in subjective sleepiness with no difference in performance on a memory search task [33]. Epidemiological studies have shown that physical activity during leisure time reduces the risk of experiencing feelings of fatigue [34]. However, more field studies involving healthcare practitioners are needed to investigate the effectiveness of these countermeasures [35]. 


\section{Fatigue proofing strategies}

We identified three types of FPS: self-regulation, task reallocation and error monitoring strategies.

Self-regulation strategies are individual controls concomitant to the task. These strategies consist of alternative routines which are considered to be more suited to the physician's current condition. Physicians' self-regulation strategies aim at reducing the demands placed upon them as the available resources grow scarce. The regulation of work procedures as a function of task demands has been investigated in air traffic control literature. According to Donath and Schulte, operators adopt load-shedding strategies to keep the workload within bearable limits and to maintain acceptable performance [36]. These strategies are changing the way the task is accomplished in a more economic way in terms of individual resources. In our study, physicians reported using alternative routines because their usual routines do not withstand poor or inconsistent performance associated with fatigue. The drawbacks of such approaches include the fact that alternative routines make the task more tedious and time-consuming. As a consequence, physicians employ alternative routines when the perceived fatigue-related risk increases beyond what is tolerable or permissible, and return to their usual and convenient routines once the perceived risk returns to an acceptable level. Self-regulation strategies point out that physicians do not perform a task the same way across contexts, but choose from multiple procedures with different associated cost and risk outcomes. Rather than teaching a single way to perform a task, training programmes should focus on teaching physicians to spot these contexts and choose the appropriate procedure.

Task re-allocation strategies are team-work adaptations which supplant the need for appropriate rest or break time. When withdrawal from the workplace is not an option, exhausted physicians reported avoiding performing tasks which are vulnerable to fatigue-related errors and to focus on less-demanding tasks. Critical tasks are redistributed within the team or to a colleague working the following shift. There are fewer deferring opportunities during the night because of a limited workforce and deferring to later is only appropriate for a narrow range of cases not requiring immediate care. As a result, task re-allocation strategies have limited application and physicians are regularly required to perform sensitive tasks while exhausted. Task re-allocation strategies also show great potential for standard protocols. The systematic identification of at-risk operators followed by a redistribution of tasks could effectively mitigate fatigue-related risk.

Error monitoring strategies are individual or team controls subsequent to the task, which aim to screen for potential fatigue-related errors. Physicians report using these strategies when they are required to perform critical tasks late in the night. In some cases, detected errors can be corrected before they develop into an adverse event. Reported error monitoring strategies include verbalising the steps involved in a task and double-checking by oneself or by others. Few studies attempted to describe the nature of error monitoring strategies, and the evidence for their effectiveness is weak [37]. Double-checking strategies have been recommended by healthcare practitioners as a technique for intercepting medication errors, but there is still insufficient evidence to support this practice [38]. Error identification and recovery is not traditionally taught or evaluated in residency training [39], and there is significant potential for such processes to be formalised. It has been demonstrated that routine monitoring of the environment was the main source of detected errors in anaesthesia [40]. Increasing standard check frequency during critical periods demonstrates great potential to mitigate fatigue-related risk. However, more research is needed to ascertain the effectiveness of specific error monitoring strategies.

\section{Conclusion and implications for safety}

Our results show that physicians have a clear picture of fatigue-related impairments and experiment with a wide range of improvisations and adjustments to manage fatiguerelated risk. Such adjustments create resilience on the local level but contribute to widening the gap between the work-as-done by practitioners and the work-as-imagined by managers. The misalignment of work-as-done and workas-imagined can make organisations more brittle, as those responsible for managing the work are unaware of the performance adjustments that are necessary to ensure patient safety [41]. It is a challenge for fatigue-related risk management to create mutually positive awareness between managers and practitioners to reduce this gap.

The focus groups proved to be an efficient tool for sharing experiences about fatigue-related risk and how to manage them. Formal workshop sessions organised throughout the year could support knowledge transfer between physicians and allow managers to identify scope for further formalisation. Mind-mapping also added significant value for generating and structuring ideas around a given subject. The implicit nature of work-as-done in hospital setting makes brainstorming tools particularly relevant. Such approaches help to elicit work perceptions and adjustments, ultimately creating mutual awareness between managers and practitioners. Workshops should also focus on informing physicians on the early signs of fatigue and how to detect them in themselves and in colleagues. Team-based proofing strategies require physicians to continuously share insights about who is at risk and which tasks are vulnerable. However, the literature shows that emergency physicians are reluctant to admit problems 
related to fatigue $[42,43]$ because of their concerns about confidentiality and what colleagues may think of their competency [44]. Hence, it is a challenge to promote a culture of safety that recognises fatigue as an unacceptable hazard and encourages physicians to disclose situations where they feel at risk.

Research about proofing strategies in residency contexts is scarce and this study adds to few pieces of the literature aiming to understand the nature of these processes. Our attempt to identify core consistencies across these strategies has resulted in a further classification of proofing strategies. Our results are bound up in the operational setting, and the strategies used by emergency physicians are likely to vary from one organisation to the other. Further research with larger samples from different facilities is encouraged to evaluate the prevalence and effectiveness of these strategies.

\section{Compliance with ethical standards}

Conflict of interest The authors declare that they have no conflict of interest.

Statement of human and animal rights All procedures performed in the study were in accordance with the ethical standards of the institutional research committee and with the 1964 Helsinki declaration and its later amendments. This article does not contain any studies with animals performed by any of the authors.

Informed consent Informed consent was obtained from all individual participants included in the study.

\section{References}

1. Williamson A, Lombardi DA, Folkard S, Stutts J, Courtney TK, Connor JL (2011) The link between fatigue and safety. Accid Anal Prev 43(2):498-515

2. Olds DM, Clarke SP (2010) The effect of work hours on adverse events and errors in health care. J Safety Res 41(2):153-162

3. Cocker F, Joss N (2016) Compassion fatigue among healthcare, emergency and community service workers: a systematic review. Int J Environ Res Public Health 13(6):618

4. Baldwin DC Jr, Daugherty SR (2004) Sleep deprivation and fatigue in residency training: results of a national survey of first- and second-year residents. Sleep 27(2):217-223

5. Folkard S (2008) Do permanent night workers show circadian adjustment? A review based on the endogenous melatonin rhythm. Chronobiol Int 25(2):215-224

6. Handel DA, Raja A, Lindsell CJ (2006) The use of sleep aids among Emergency Medicine residents: a web based survey. BMC Health Services Research 6:136

7. Tür FÇ, Toker I, Tür B, Hacar S (2015) Assessment of the Pittsburgh sleep quality index among Physician's speciality Who work night shifts. Emerg Med Open J. 1:5-11

8. Dawson D, McCulloch K (2005) Managing fatigue: it's about sleep. Sleep Med Rev 9(5):365-380

9. Dawson D, Chapman J, Thomas MJW (2012) Fatigue- proofing: a new approach to reducing fatigue-related risk using the principles of error management. Sleep Med Rev 16:167-175

10. Mayring P (2014) Qualitative content analysis: theoretical background and procedures, approaches to qualitative research in mathematics education. Norma Presmeg, Illinois, pp 365-380

11. Helmreich R, Musson D, Sexton J (2004) Human factors and safety in surgery. In: Nora PF, Manuel B (eds) Surgical patient safety essential information for surgeons in today's environment. American College of Surgeons, Chicago

12. Harrison Y, Horne JA (2000) Sleep loss and temporal memory. Q J Exp Psychol A 53:271-279

13. Alhola P, Polo-Kantola P (2007) Sleep deprivation: impact on cognitive performance. Neuropsychiatr Dis Treat. 3(5):553-567

14. Schnyer DM, Zeithamova D, Williams V (2009) Decision-making under conditions of sleep deprivation: cognitive and neural consequences. Milit Psychol 21(Suppl 1):S36-S45

15. Khazaie H, Tahmasian M, Ghadami MR, Safaei H, Ekhtiari H, Samadzadeh S, Schwebel DC, Russo MB (2010) The effects of chronic partial sleep deprivation on cognitive functions of medical residents. Iran J Psychiatry 5(2):74-77

16. Centers for Disease Control and Prevention. Chronic fatigue syndrome. https://www.cdc.gov/me-cfs/treatment/index.html. Accessed 21 Dec 2017

17. Coetzee SK, Klopper HC (2010) Compassion fatigue within nursing practice: a concept analysis. Nurs Health Sci. 12(2):235-243

18. Jeanmonod R, Brook C, Winther M, Pathak S, Boyd M (2009) Resident productivity as a function of emergency department volume, shift time of day, and cumulative time in the emergency department. Am J Emerg Med 27(3):313-319

19. Sexton JB, Thomas EJ, Helmreich RL (2000) Error, stress, and teamwork in medicine and aviation: cross sectional surveys. BMJ 320(7237):745-749

20. Passalacqua SA, Segri C (2012) The effect of resident physician stress, burnout, and empathy on patient-centered communication during the long-call shift. Health Commun. 27:449-456

21. Friesen LD, Vidyarthi Baron RB, Katz PP (2008) Factors associated with intern fatigue. J Gen Intern Med 23(12):1981-1986

22. Singh H, Thomas EJ, Petersen LA, Studdert DM (2007) Medical errors involving trainees: a study of closed malpractice claims from 5 insurers. Arch Intern Med 167(19):2030-2036

23. West CP, Tan AD, Habermann TM, Sloan JA, Shanafelt TD (2009) Association of resident fatigue and distress with perceived medical errors. JAMA 302(12):1294-1300

24. Woolf SH, Kuzel AJ, Dovey SM, Phillips RL (2004) A string of mistakes: the importance of cascade analysis in describing, counting, and preventing medical errors. Ann Fam Med 2(4):317-326

25. Khan A, Rathore AW (2016) Medical errors; causes, consequences, emotional response and resulting behavioral change. Pak J Med Sci 32(3):523-528

26. Driskell JE, Mullen B (2005) The efficacy of naps as a fatigue countermeasure: a meta-analytic integration. Hum Factors 2:360-377

27. Hilditch CJ, Stephanie A, Dorrian J, Banks S (2016) A 30-minute, but not a 10-minute nighttime nap is associated with sleep inertia. Sleep 39(3):675-685

28. Berry BB, Wagner MH (2015) In Sleep medicine pearls, 3rd edn. Elsevier, Florida

29. Reyner LA, Horne JA (1997) Suppression of sleepiness in drivers: combination of caffeine with a short nap. Psychophysiology 34(6):721-725

30. Sack RL, Auckley D, Auger RR, Carskadon MA, Wright KP Jr, Vitiello MV, Zhdanova IV (2007) Circadian rhythm sleep disorders: part I, basic principles, shift work and jet lag disorders. An American Academy of Sleep Medicine review. Sleep 30(11):1460-1483 
31. Danziger S, Levav J, Avnaim-Pesso L (2001) Extraneous factors in judicial decisions Proc Natl Acad Sci 108(17):6889-6892

32. Puddester D (2014) Managing and mitigating fatigue in the era of changing resident duty hours. BMC Med Educ 14(Suppl 1):S3

33. Hayashi M, Masuda A, Hori T (2003) The alerting effects of caffeine, bright light and face washing after a short daytime nap. Clin Neurophysiol 114(12):2268-2278

34. Puetz TW, O'Connor PJ, Dishman RK (2006) Effects of chronic exercise on feelings of energy and fatigue: a quantitative synthesis. Psychol Bull 132(6):866-876

35. Hilditch CJ, Dorrian J, Banks S (2016) Time to wake up: reactive countermeasures to sleep inertia. Ind Health 54(6):528-541

36. Schulte A, Donath D (2011) Measuring self-adaptive UAV operators' load-shedding strategies under high workload. Eng Psychol Cognit Ergon 13:342-351

37. Hodgkinson B, Koch S, Nay R, Nichols K (2006) Strategies to reduce medication errors with reference to older adults. Int J Evid Based Healthc. 4(1):2-41

38. Alsulami Z, Conroy S, Choonara I (2012) Double checking the administration of medicines: what is the evidence? A systematic review. Arch Dis Child 97(9):833-837
39. Sternbach JM, Wang K, El Khoury R, Teitelbaum EN, Meyerson SL (2016) Measuring error identification and recovery skills in surgical residents presented at the fifty-second annual meeting of the society of thoracic surgeons, phoenix, AZ, Jan 23-27

40. Nyssen AS, Blavier A (2006) Error detection: a study in anaesthesia. Ergonomics 49:517-525

41. Sujan MA, Pozzi S, Valbonesi C (2016) Reporting and learning: from extraordinary to ordinary. Resilient Health Care, vol 3: reconciling work-as-imagined and work-as-done

42. Kay M, Mitchell G, Clavarino A, Doust J (2008) Doctors as patients: a systematic review of doctors' health access and the barriers they experience. Br J Gen Pract 58(552):501-508

43. O'Reilly K (2012) Stressed physicians reluctant to seek support. In Browser KJ, Riba MB. Physician mental health and well-being: research and practice. http://www.amed-news.com/apps/pbcs.dll/ personalia?ID=koreilly. Accessed 17 Dec 2017

44. Rosenstein AH (2011) Managing disruptive behaviors in the health care setting: focus on obstetrics services. Am J Obstet Gynecol 204:187-192 